\title{
Approximation of the Set of Trajectories of the Nonlinear Control System with Limited Control Resources
}

\author{
Nesir Huseyin ${ }^{a}$, Anar Huseyin ${ }^{b}$ and Khalik Guseinov ${ }^{c}$ \\ ${ }^{a}$ Cumhuriyet University, Faculty of Education, Department of Mathematics \\ and Science Education \\ 58140 Sivas, Turkey \\ ${ }^{b}$ Cumhuriyet University, Faculty of Science, Department of Statistics \\ 58140 Sivas, Turkey \\ ${ }^{c}$ Anadolu University, Faculty of Science, Department of Mathematics \\ 26470 Eskisehir, Turkey \\ E-mail(corresp.): kguseynov@anadolu.edu.tr \\ E-mail: nhuseyin@cumhuriyet.edu.tr \\ E-mail: ahuseyin@cumhuriyet.edu.tr
}

Received June 22, 2017; revised December 6, 2017; accepted December 7, 2017

\begin{abstract}
In this paper the control system described by a Urysohn type integral equation is studied. It is assumed that the control functions have integral constraint. Approximation of the set of trajectories generated by all admissible control functions is considered. Step by step way, the set of admissible control functions is replaced by a set consisting of a finite number of control functions which generates a finite number of trajectories. An evaluation of the Hausdorff distance between the set of trajectories of the system and the set, consisting of a finite number of trajectories is obtained.
\end{abstract}

Keywords: control system, nonlinear integral equation, integral constraint, set of trajectories, approximation.

AMS Subject Classification: 45G15; 49M25; 93C10.

\section{Introduction}

Control systems with integral constraints on control functions arise in many problems of theory and applications. If the control effort is exhausted by consumption, such as energy, fuel, finance and food, then integral constraint on

This is an Open Access article distributed under the terms of the Creative Commons Attribution License (http://creativecommons.org/licenses/by/4.0/), which permits unrestricted use, distribution, and reproduction in any medium, provided the original author and source are credited. 
the control functions is inevitable. For example the motion of the flying object with variable mass is described by the control system with integral constraint on the control functions (see, e.g. [8], [20]). One of the important notion of the control systems theory is the set of trajectories, generated by all admissible control functions. Knowing the set of trajectories allows to forecast different behaviors of the control system and design the control functions with desired properties (see, e.g. [21]). Various topological properties and approximation of the set of trajectories of the control system described by an ordinary differential equation with integral constraint on the control functions are studied in papers [7], [10], [11], [18], [22], [23].

The mathematical models of many processes in mechanics, physics, economy, biology are described via nonlinear integral equations (see, e.g. [2], [5], [9], [19], [24], [25] and references therein). Pointing out the importance of the integral equations, W. Heisenberg in his well known "Physics and Philosophy" writes: "The final equation of motion for matter will probably be some quantized nonlinear wave equation... This wave equation will probably be equivalent to rather complicated sets of integral equations..." (see, [12], page 68). It should be noted that the theory of integral equations is considered one of the origins of contemporary functional analysis (see, e.g. [13], chapter 1, page 2).

It is known that the solutions of the initial and boundary value problems for differential equations can be expressed via solutions of the appropriate integral equations (see, e.g. [9], [13], [19]).

Control systems described by the integral equations with geometric constraints on the controls are discussed in [1], [3], [4], [6]. In [14], [16], [15], [17] approximation and topological properties of the set of trajectories of the control systems described by nonlinear integral equations with integral constraints on the control functions are considered. In this paper the control system described by a Urysohn type integral equation with integral constraint on the control functions is studied, where the trajectory of the system is multivariable continuous function. The closed ball of the space $L_{p}, p>1$, is chosen as the set of admissible control functions. An approximation of the set of trajectories of the system, generated by all admissible control functions is given. Step by step way, the set of control functions is replaced by a set, consisting of a finite number of control functions which generates a finite number of trajectories. An evaluation of the Hausdorff distance between the set of trajectories of the system and the set, consisting of a finite number of trajectories, is obtained.

The paper is organized as follows: In Section 2 the basic conditions which satisfy the system's equation are given and some auxiliary propositions which are used in following arguments, are formulated. In Section 3 the set of admissible control functions is narrowed down and new set, consisting of integral and geometric constrained control functions is defined. An estimation of the Hausdorff distance between the set of trajectories of the system and the set of trajectories generated by the mixed constrained control functions is obtained (Proposition 6). In Section 4 the set of mixed constrained and piecewise constant control functions is introduced. The Hausdorff distance between the set of trajectories generated by the mixed constrained control functions and the set of trajectories, generated by the mixed constrained and piecewise constant 
control functions, is evaluated (Proposition 7). In Section 5 the Hausdorff distance between the set of trajectories generated by the mixed constrained and piecewise constant control functions and the set of trajectories, generated by the mixed constrained and piecewise constant control functions with norms from a uniform mesh, is estimated (Proposition 8). In Section 6 the set mixed constrained and piecewise constant control functions with norms from a uniform mesh, is replaced by a set consisting of a finite number of control functions which generates a finite number of trajectories. An estimation of the Hausdorff distance between the set of trajectories, generated by the mixed constrained and piecewise constant control functions with norms from a uniform mesh and the set consisting of a finite number of trajectories is given (Proposition 9). Concluding the results, obtained in previous sections, in Section 7 the main result of the paper is formulated. The Hausdorff distance between the set of trajectories of the system and the set consisting of a finite number of trajectories is evaluated (Theorem 1).

\section{System's behaviour}

Consider the control system described by a Urysohn type integral equation

$$
x(\xi)=f(\xi, x(\xi))+\lambda \int_{E}\left[K_{1}(\xi, s, x(s))+K_{2}(\xi, s, x(s)) u(s)\right] d s,
$$

where $x(s) \in \mathbb{R}^{n}$ is the state vector, $u(s) \in \mathbb{R}^{m}$ is the control vector, $(\xi, s) \in$ $E \times E, E \subset \mathbb{R}^{k}$ is a compact set, $\lambda \in \mathbb{R}$.

For given $p>1$ and $r>0$ the set

$$
U_{p, r}=\left\{u(\cdot) \in L_{p}\left(E ; \mathbb{R}^{m}\right):\|u(\cdot)\|_{p} \leq r\right\}
$$

is called the set of admissible control functions and each $u(\cdot) \in U_{p, r}$ is said to be an admissible control function, where $L_{p}\left(E ; \mathbb{R}^{m}\right)$ is the space of Lebesgue measurable functions $u(\cdot): E \rightarrow \mathbb{R}^{m}$ such that $\|u(\cdot)\|_{p}<+\infty,\|u(\cdot)\|_{p}=$ $\left(\int_{E}\|u(s)\|^{p} d s\right)^{\frac{1}{p}},\|\cdot\|$ denotes the Euclidean norm.

It is assumed that the functions $f(\cdot), K_{1}(\cdot), K_{2}(\cdot)$ and real number $\lambda$ satisfy the following conditions:

A. The functions $f(\cdot): E \times \mathbb{R}^{n} \rightarrow \mathbb{R}^{n}, K_{1}(\cdot): E \times E \times \mathbb{R}^{n} \rightarrow \mathbb{R}^{n}$ and $K_{2}(\cdot): E \times E \times \mathbb{R}^{n} \rightarrow \mathbb{R}^{n \times m}$ are continuous;

B. There exist $l_{0} \in[0,1), l_{1}>0$ and $l_{2}>0$ such that

$$
\left\|f\left(\xi, x_{2}\right)-f\left(\xi, x_{1}\right)\right\| \leq l_{0}\left\|x_{2}-x_{1}\right\|
$$

for every $\left(\xi, x_{1}\right) \in E \times \mathbb{R}^{n},\left(\xi, x_{2}\right) \in E \times \mathbb{R}^{n}$ and

$$
\begin{aligned}
\left\|K_{1}\left(\xi, s, x_{2}\right)-K_{1}\left(\xi, s, x_{1}\right)\right\| & \leq l_{1}\left\|x_{2}-x_{1}\right\|, \\
\left\|K_{2}\left(\xi, s, x_{2}\right)-K_{2}\left(\xi, s, x_{1}\right)\right\| & \leq l_{2}\left\|x_{2}-x_{1}\right\|
\end{aligned}
$$

for every $\left(\xi, s, x_{1}\right) \in E \times E \times \mathbb{R}^{n}$ and $\left(\xi, s, x_{2}\right) \in E \times E \times \mathbb{R}^{n}$; 
C. The inequality $0 \leq \lambda\left[l_{1} \mu(E)+l_{2}[\mu(E)]^{\frac{p-1}{p}} r\right]<1-l_{0}$ is satisfied, where $\mu(E)$ denotes the Lebesgue measure of the set $E$.

Denote

$$
l(\lambda)=l_{0}+\lambda\left(l_{1} \mu(E)+l_{2}[\mu(E)]^{\frac{p-1}{p}} r\right) .
$$

Let $u(\cdot) \in U_{p, r}$. A continuous function $x(\cdot): E \rightarrow \mathbb{R}^{n}$ satisfying the equation (2.1) for every $\xi \in E$ is said to be a trajectory of the system (2.1), generated by the admissible control function $u(\cdot)$. The set of all trajectories of the system (2.1) generated by all admissible control functions $u(\cdot) \in U_{p, r}$ is denoted by $\mathbf{X}_{p, r}$.

The conditions $A-C$ guarantee that every admissible control function $u(\cdot) \in$ $U_{p, r}$ generates a unique trajectory of the system (2.1) (see, [16]). Now let us give some auxiliary propositions which will be used in following arguments.

Proposition 1. [16] Let $E \subset \mathbb{R}^{k}$ be a compact set, $v(\cdot): E \rightarrow \mathbb{R}$ and $g(\cdot)$ : $E \rightarrow \mathbb{R}$ be continuous functions, $\psi(\cdot): E \rightarrow[0,+\infty)$ be a Lebesgue integrable function, $\int_{E} \psi(s) d s<1$ and

$$
v(\xi) \leq g(\xi)+\int_{E} \psi(s) v(s) d s
$$

for every $\xi \in E$. Then the inequality

$$
v(\xi) \leq g(\xi)+\int_{E} g(s) \psi(s) d s /\left(1-\int_{E} \psi(s) d s\right)
$$

holds for every $\xi \in E$. Moreover, if $\int_{E} \psi(s) d s=a_{0}<1, g(\xi)=g_{*}$ for every $\xi \in E$, then it follows from (2.3) that $v(\xi) \leq g_{*} /\left(1-a_{0}\right)$ for every $\xi \in E$.

The following propositions characterize compactness property of the set of trajectories.

Proposition 2. [16] The set of trajectories $\mathbf{X}_{p, r}$ of system (2.1) is a bounded subset of the space $C\left(E ; \mathbb{R}^{n}\right)$, i.e. there exists $\gamma_{*}>0$ such that

$$
\|x(\cdot)\|_{C} \leq \gamma_{*}
$$

for every $x(\cdot) \in \mathbf{X}_{p, r}$. Here $C\left(E ; \mathbb{R}^{n}\right)$ is the space of continuous functions $x(\cdot): E \rightarrow \mathbb{R}^{n}$ with norm $\|x(\cdot)\|_{C}=\max \{\|x(\xi)\|: \xi \in E\}$.

Let $\Delta>0$ be a given number, $\gamma_{*}>0$ be defined by (2.4),

$$
\begin{aligned}
& B_{n}\left(\gamma_{*}\right)=\left\{x \in \mathbb{R}^{n}:\|x\| \leq \gamma_{*}\right\}, \quad B_{C}(1)=\left\{x(\cdot) \in C\left(E ; \mathbb{R}^{n}\right):\|x(\cdot)\|_{C} \leq 1\right\}, \\
& D_{1}=E \times B_{n}\left(\gamma_{*}\right), \quad D_{2}=E \times E \times B_{n}\left(\gamma_{*}\right), \\
& M_{2}=\max \left\{\left\|K_{2}(\xi, s, x)\right\|:(\xi, s, x) \in D_{2}\right\}, \\
& \omega_{0}(\Delta)=\max \left\{\left\|f\left(\xi_{2}, x\right)-f\left(\xi_{1}, x\right)\right\|:\left\|\xi_{2}-\xi_{1}\right\| \leq \Delta,\right. \\
& \left.\qquad\left(\xi_{1}, x\right) \in D_{1}, \quad\left(\xi_{2}, x\right) \in D_{1}\right\}, \\
& \omega_{1}(\Delta)=\max \left\{\left\|K_{1}\left(\xi_{2}, s_{2}, x_{2}\right)-K_{1}\left(\xi_{1}, s_{1}, x_{1}\right)\right\|:\left\|\xi_{2}-\xi_{1}\right\| \leq \Delta,\right. \\
& \left.\left\|s_{2}-s_{1}\right\| \leq \Delta, \quad\left\|x_{2}-x_{1}\right\| \leq \Delta, \quad\left(\xi_{1}, s_{1}, x_{1}\right) \in D_{2}, \quad\left(\xi_{2}, s_{2}, x_{2}\right) \in D_{2}\right\}
\end{aligned}
$$




$$
\begin{aligned}
& \omega_{2}(\Delta)=\max \left\{\left\|K_{2}\left(\xi_{2}, s_{2}, x_{2}\right)-K_{2}\left(\xi_{1}, s_{1}, x_{1}\right)\right\|:\left\|\xi_{2}-\xi_{1}\right\| \leq \Delta,\right. \\
& \left.\left\|s_{2}-s_{1}\right\| \leq \Delta, \quad\left\|x_{2}-x_{1}\right\| \leq \Delta, \quad\left(\xi_{1}, s_{1}, x_{1}\right) \in D_{2}, \quad\left(\xi_{2}, s_{2}, x_{2}\right) \in D_{2}\right\}, \\
& \varphi(\Delta)=\frac{1}{1-l_{0}}\left\{\omega_{0}(\Delta)+\lambda \mu(E) \omega_{1}(\Delta)+\lambda \omega_{2}(\Delta)[\mu(E)]^{\frac{p-1}{p}} r\right\}, \\
& \varphi_{*}(\Delta)=\max \{\Delta, \varphi(\Delta)\} .
\end{aligned}
$$

It is obvious that the functions $\varphi(\cdot):(0,+\infty) \rightarrow[0,+\infty)$ and $\varphi_{*}(\cdot)$ : $(0,+\infty) \rightarrow(0,+\infty)$ are not decreasing and $\varphi(\Delta) \rightarrow 0^{+}, \varphi_{*}(\Delta) \rightarrow 0^{+}$as $\Delta \rightarrow 0^{+}$.

Proposition 3. [16] For every $x(\cdot) \in \mathbf{X}_{p, r}, \xi_{1} \in E, \xi_{2} \in E$ the inequality

$$
\left\|x\left(\xi_{2}\right)-x\left(\xi_{1}\right)\right\| \leq \varphi\left(\left\|\xi_{2}-\xi_{1}\right\|\right)
$$

holds, where $\varphi(\cdot)$ is defined by (2.7).

Proposition 4. [16] The set of trajectories $\mathbf{X}_{p, r}$ of the system (2.1) is a compact subset of the space $C\left(E ; \mathbb{R}^{n}\right)$.

Denote

$$
q_{*}=M_{2} \lambda /(1-l(\lambda)),
$$

where $l(\lambda)$ is defined by relation (2.2), $M_{2}$ is defined by relation (2.5).

Proposition 5. Let $x_{1}(\cdot) \in \mathbf{X}_{p, r}$ and $x_{2}(\cdot) \in \mathbf{X}_{p, r}$ be trajectories of the system (2.1) generated by the admissible control functions $u_{1}(\cdot) \in U_{p, r}$ and $u_{2}(\cdot) \in U_{p, r}$ respectively. Then

$$
\left\|x_{1}(\xi)-x_{2}(\xi)\right\| \leq q_{*} \int_{E}\left\|u_{1}(s)-u_{2}(s)\right\| d s
$$

for every $\xi \in E$.

The validity of the proposition follows from conditions $A-C$ and Proposition 1.

\section{Geometric constraints}

Define new set of control functions. For $H \in(0, \infty)$ we set

$$
U_{p, r}^{H}=\left\{u(\cdot) \in U_{p, r}:\|u(\xi)\| \leq H \text { for every } \xi \in E\right\}
$$

and let $\mathbf{X}_{p, r}^{H}$ be the set of trajectories of the system (2.1) generated by the control functions $u(\cdot) \in U_{p, r}^{H}$. We denote

$$
\kappa_{*}=2 q_{*} r^{p}
$$

where $q_{*}$ is defined by $(2.9)$. 
The Hausdorff distance between the sets $Y \subset C\left(E ; \mathbb{R}^{n}\right)$ and $W \subset C\left(E ; \mathbb{R}^{n}\right)$ is denoted by $h_{C}(Y, W)$ and is defined as

$$
h_{C}(Y, W)=\max \left\{\sup _{y(\cdot) \in Y} d_{C}(y(\cdot), W), \sup _{w(\cdot) \in W} d_{C}(w(\cdot), Y)\right\},
$$

where $d_{C}(y(\cdot), W)=\inf \left\{\|y(\cdot)-w(\cdot)\|_{C}: w(\cdot) \in W\right\}$ is the distance between the point $y(\cdot)$ and the set $W$.

Proposition 6. For every $H \in(0, \infty)$ the inequality

$$
h_{C}\left(\mathbf{X}_{p, r}, \mathbf{X}_{p, r}^{H}\right) \leq \kappa_{*} / H^{p-1}
$$

is satisfied.

Proof. Let $x_{*}(\cdot) \in \mathbf{X}_{p, r}$ be an arbitrary trajectory of the system (2.1) generated by the control function $u_{*}(\cdot) \in U_{p, r}$. Define new control function $u_{0}(\cdot)$ : $E \rightarrow \mathbb{R}^{m}$ setting

$$
u_{0}(s)= \begin{cases}u_{*}(s), & \text { if }\left\|u_{*}(s)\right\| \leq H, \\ H \frac{u_{*}(s)}{\left\|u_{*}(s)\right\|}, & \text { if }\left\|u_{*}(s)\right\|>H,\end{cases}
$$

where $s \in E$. It is not difficult to verify that $u_{0}(\cdot) \in U_{p, r}^{H}$. Let $x_{0}(\cdot)$ be the trajectory of the system $(2.1)$ generated by the control function $u_{0}(\cdot) \in U_{p, r}^{H}$ and $G=\left\{\xi \in E:\left\|u_{*}(s)\right\|>H\right\}$. Then $x_{0}(\cdot) \in \mathbf{X}_{p, r}^{H}$ and Proposition 5 implies that

$$
\left\|x_{*}(\xi)-x_{0}(\xi)\right\| \leq q_{*} \int_{G}\left\|u_{*}(s)-u_{0}(s)\right\| d s
$$

for every $\xi \in E$. Inclusion $u_{*}(\cdot) \in U_{p, r}$ yields that

$$
H^{p} \cdot \mu(G) \leq \int_{G}\left\|u_{*}(s)\right\|^{p} d s \leq \int_{E}\left\|u_{*}(s)\right\|^{p} d s \leq r^{p},
$$

and hence

$$
\mu(G) \leq r^{p} / H^{p} .
$$

Since $u_{*}(\cdot) \in U_{p, r}$ and $u_{0}(\cdot) \in U_{p, r}^{H}$, then from (3.1)-(3.3) and Hölder's inequality it follows

$$
\left\|x_{*}(\xi)-x_{0}(\xi)\right\| \leq 2 q_{*} r[\mu(G)]^{\frac{p-1}{p}} \leq \frac{2 q_{*} r^{p}}{H^{p-1}}=\frac{\kappa_{*}}{H^{p-1}}
$$

for every $\xi \in E$, and consequently

$$
\left\|x_{*}(\cdot)-x_{0}(\cdot)\right\|_{C} \leq \frac{\kappa_{*}}{H^{p-1}} .
$$

Since $x_{*}(\cdot) \in \mathbf{X}_{p, r}$ is an arbitrarily chosen trajectory, then we have from (3.4) that

$$
\mathbf{X}_{p, r} \subset \mathbf{X}_{p, r}^{H}+\frac{\kappa_{*}}{H^{p-1}} B_{C}(1) .
$$

The inclusion $\mathbf{X}_{p, r}^{H} \subset \mathbf{X}_{p, r}$ and (3.5) complete the proof. 


\section{Piecewise constant control functions}

Let us define a $\Delta$-partition of the given compact set $E \subset \mathbb{R}^{k}$. Denote $B_{k}^{\circ}(\xi, \eta)=$ $\left\{y \in \mathbb{R}^{k}:\|y-\xi\|<\eta\right\}$. It is known that

$$
\mu\left(B_{k}^{\circ}(\xi, \eta)\right)=\frac{\pi^{k / 2}}{\Gamma(k / 2+1)} \eta^{k},
$$

where $\Gamma(\cdot)$ is Euler's function. Let $\Delta>0$ be a given number and

$$
\eta \leq \min \left\{\frac{\Delta}{2},\left(\frac{\Gamma(k / 2+1)}{\pi^{k / 2}} \Delta\right)^{1 / k}\right\} .
$$

Then (4.1) and (4.2) imply that

$$
\eta \leq \frac{\Delta}{2}, \quad \mu\left(B_{k}^{\circ}(\xi, \eta)\right) \leq \Delta .
$$

It is obvious that $E \subset \bigcup_{\xi \in E} B_{k}^{\circ}(\xi, \eta)$. Since the set $E \subset \mathbb{R}^{k}$ is a compact set, then there exist $B_{k}^{\circ}\left(\xi_{1}, \eta\right), B_{k}^{\circ}\left(\xi_{2}, \eta\right), \ldots, B_{k}^{\circ}\left(\xi_{N}, \eta\right)$ such that

$$
E \subset \bigcup_{i=1}^{N} B_{k}^{\circ}\left(\xi_{i}, \eta\right) .
$$

Denote $B_{k}^{*}\left(\xi_{i}, \eta\right)=E \cap B_{k}^{\circ}\left(\xi_{i}, \eta\right)$. From (4.3) and (4.4) it follows that the sets $B_{k}^{*}\left(\xi_{i}, \eta\right), i=1,2, \ldots, N$ are open sets with respect to the topology induced in $E$,

$$
B_{k}^{*}\left(\xi_{i}, \eta\right) \subset B_{k}^{\circ}\left(\xi_{i}, \eta\right), \quad \mu\left(B_{k}^{*}\left(\xi_{i}, \eta\right)\right) \leq \Delta
$$

for every $i=1,2, \ldots, N$ and

$$
E=\bigcup_{i=1}^{N} B_{k}^{*}\left(\xi_{i}, \eta\right) .
$$

We set $E_{1}=B_{k}^{*}\left(\xi_{1}, \eta\right), E_{2}=B_{k}^{*}\left(\xi_{2}, \eta\right) \backslash B_{k}^{*}\left(\xi_{1}, \eta\right), \ldots, E_{j}=B_{k}^{*}\left(\xi_{j}, \eta\right) \backslash$ $\bigcup_{i=1}^{j-1} B_{k}^{*}\left(\xi_{i}, \eta\right), \ldots, E_{N}=B_{k}^{*}\left(\xi_{N}, \eta\right) \backslash \bigcup_{i=1}^{N-1} B_{k}^{*}\left(\xi_{i}, \eta\right)$. Without loss of generality let us assume that $E_{i} \neq \emptyset$ for every $i=1,2, \ldots, N$. (4.5) and (4.6) imply that the sets $E_{i} \subset E, i=1,2, \ldots, N$ are Lebesgue measurable,

$$
E_{i} \subset B_{k}^{*}\left(\xi_{i}, \eta\right) \subset B_{k}^{\circ}\left(\xi_{i}, \eta\right), \quad \mu\left(E_{i}\right) \leq \Delta
$$

for every $i=1,2, \ldots, N$,

$$
E_{i} \bigcap E_{j}=\emptyset
$$

for every $i=1,2, \ldots, N$ and $j=1,2, \ldots, N$ such that $i \neq j$ and

$$
E=\bigcup_{i=1}^{N} E_{i}
$$


The collection of sets $\Gamma=\left\{E_{1}, E_{2}, \ldots, E_{N}\right\}$ satisfying the relations (4.7)(4.9) is called a finite $\Delta$-partition of the compact set $E \subset \mathbb{R}^{k}, \Delta>0$ is said to be the diameter of the partition $\Gamma$.

Now for given $\Delta>0$, compact set $E \subset \mathbb{R}^{k}$ and its finite $\Delta$-partition $\Gamma=\left\{E_{1}, E_{2} \ldots, E_{N}\right\}$ we define new set of control functions consisting of mixed constrained and piecewise constant control functions, setting

$$
U_{p, r}^{H, \Delta}=\left\{u(\cdot) \in U_{p, r}^{H}: u(\xi)=u_{i} \text { for every } \xi \in E_{i}, i=1,2, \ldots, N\right\} .
$$

By $\mathbf{X}_{p, r}^{H, \Delta}$ we denote the set of trajectories of the control system (2.1) generated by the control functions $u(\cdot) \in U_{p, r}^{H, \Delta}$ and let

$$
\beta_{*}(\Delta)=\frac{2 \lambda \omega_{2}\left(\varphi_{*}(\Delta)\right)[\mu(E)]^{\frac{p-1}{p}} r}{1-l(\lambda)},
$$

where $l(\lambda), \omega_{2}(\cdot)$ and $\varphi_{*}(\cdot)$ are defined by $(2.2),(2.6)$ and $(2.8)$ respectively.

The following proposition characterizes the Hausdorff distance between the sets $\mathbf{X}_{p, r}^{H}$ and $\mathbf{X}_{p, r}^{H, \Delta}$.

Proposition 7. For every $H \in(0, \infty)$ and finite $\Delta$-partition $\Gamma=\left\{E_{1}, E_{2}, \ldots\right.$, $\left.E_{N}\right\}$ of the compact set $E \subset \mathbb{R}^{k}$, the inequality

$$
h_{C}\left(\mathbf{X}_{p, r}^{H}, \mathbf{X}_{p, r}^{H, \Delta}\right) \leq \beta_{*}(\Delta)
$$

is verified.

Proof. Choose an arbitrary trajectory $x_{*}(\cdot) \in \mathbf{X}_{p, r}^{H}$ of the system (2.1) generated by the control function $u_{*}(\cdot) \in U_{p, r}^{H}$ and define new control function $\tilde{u}(\cdot): E \rightarrow \mathbb{R}^{m}$ setting

$$
\tilde{u}(\xi)=\frac{1}{\mu\left(E_{i}\right)} \int_{E_{i}} u_{*}(s) d s, \xi \in E_{i}, i=1,2, \ldots, N .
$$

Since $\left\|u_{*}(s)\right\| \leq H$ for every $s \in E$, then (4.11) implies that

$$
\|\tilde{u}(s)\| \leq H \text { for every } s \in E .
$$

Further, it follows from (4.11) and Hölder's inequality that

$$
\|\tilde{u}(\xi)\| \leq \frac{1}{\left[\mu\left(E_{i}\right)\right]^{\frac{1}{p}}}\left(\int_{E_{i}}\left\|u_{*}(s)\right\|^{p} d s\right)^{\frac{1}{p}}, \xi \in E_{i}, i=1,2, \ldots, N
$$

and hence

$$
\int_{E_{i}}\|\tilde{u}(s)\|^{p} d s \leq \int_{E_{i}}\left\|u_{*}(s)\right\|^{p} d s
$$

for every $i=1,2, \ldots, N$. Since $\left\|u_{*}(\cdot)\right\|_{p} \leq r$, then the last inequality implies that

$$
\begin{aligned}
\int_{E}\|\tilde{u}(s)\|^{p} d s & =\sum_{i=1}^{N} \int_{E_{i}}\|\tilde{u}(s)\|^{p} d s \\
& \leq \sum_{i=1}^{N} \int_{E_{i}}\left\|u_{*}(s)\right\|^{p} d s=\int_{E}\left\|u_{*}(s)\right\|^{p} d s \leq r^{p} .
\end{aligned}
$$


From (4.11)-(4.13) it follows that $\tilde{u}(\cdot) \in U_{p, r}^{H, \Delta}$. Let $\tilde{x}(\cdot)$ be the trajectory of the system $(2.1)$ generated by the control function $\tilde{u}(\cdot)$. Then $\tilde{x}(\cdot) \in \mathbf{X}_{p, r}^{H, \Delta}$ and according to the Condition $B$ we have that

$$
\begin{aligned}
\left\|x_{*}(\xi)-\tilde{x}(\xi)\right\| \leq & \frac{\lambda}{1-l_{0}} \int_{E}\left[l_{1}+l_{2}\|\tilde{u}(s)\|\right]\left\|x_{*}(s)-\tilde{x}(s)\right\| d s \\
& +\frac{\lambda}{1-l_{0}}\left\|\int_{E} K_{2}\left(\xi, s, x_{*}(s)\right)\left[u_{*}(s)-\tilde{u}(s)\right] d s\right\| \\
= & \frac{\lambda}{1-l_{0}} \int_{E}\left[l_{1}+l_{2}\|\tilde{u}(s)\|\right]\left\|x_{*}(s)-\tilde{x}(s)\right\| d s \\
& +\frac{\lambda}{1-l_{0}}\left\|\sum_{i=1}^{N} \int_{E_{i}} K_{2}\left(\xi, s, x_{*}(s)\right)\left[u_{*}(s)-\tilde{u}(s)\right] d s\right\|
\end{aligned}
$$

for every $\xi \in E$. By virtue of (4.11) we have that

$$
\int_{E_{i}} \tilde{u}(s) d s=\int_{E_{i}} u_{*}(s) d s
$$

for every $i=1,2, \ldots, N$. Let $\xi \in E$ and $i=1,2, \ldots, N$ be fixed. Now let us choose an arbitrary $s_{i} \in E_{i}$. Then (4.15) yields that

$$
\begin{aligned}
\| \int_{E_{i}} & K_{2}\left(\xi, s, x_{*}(s)\right)\left[u_{*}(s)-\tilde{u}(s)\right] d s \| \\
\quad= & \left\|\int_{E_{i}}\left[K_{2}\left(\xi, s, x_{*}(s)\right)-K_{2}\left(\xi, s_{i}, x_{*}\left(s_{i}\right)\right)\right]\left[u_{*}(s)-\tilde{u}(s)\right] d s\right\| \\
\quad \leq & \int_{E_{i}}\left\|K_{2}\left(\xi, s, x_{*}(s)\right)-K_{2}\left(\xi, s_{i}, x_{*}\left(s_{i}\right)\right)\right\|\left\|u_{*}(s)-\tilde{u}(s)\right\| d s .
\end{aligned}
$$

Since $s_{i} \in E_{i}$ then from (2.8), (4.2) and (4.7) it follows that

$$
\left\|s-s_{i}\right\| \leq \Delta \leq \varphi_{*}(\Delta)
$$

for every $s \in E_{i}$. (2.8), (4.17) and Proposition 3 imply that

$$
\left\|x_{*}(s)-x_{*}\left(s_{i}\right)\right\| \leq \varphi(\Delta) \leq \varphi_{*}(\Delta)
$$

for every $s \in E_{i}$. Thus, from (2.6) and (4.16)-(4.18) it follows that

$$
\left\|\int_{E_{i}} K_{2}\left(\xi, s, x_{*}(s)\right)\left[u_{*}(s)-\tilde{u}(s)\right] d s\right\| \leq \omega_{2}\left(\varphi_{*}(\Delta)\right) \int_{E_{i}}\left\|u_{*}(s)-\tilde{u}(s)\right\| d s .
$$

Since $u_{*}(\cdot) \in U_{p, r}^{H}$ and $\tilde{u}(\cdot) \in U_{p, r}^{H, \Delta}$, then (4.19) and Hölder's inequality imply that

$$
\begin{aligned}
& \left\|\sum_{i=1}^{N} \int_{E_{i}} K_{2}\left(\xi, s, x_{*}(s)\right)\left[u_{*}(s)-\tilde{u}(s)\right] d s\right\| \\
& \quad \leq \omega_{2}\left(\varphi_{*}(\Delta)\right) \sum_{i=1}^{N} \int_{E_{i}}\left\|u_{*}(s)-\tilde{u}(s)\right\| d s
\end{aligned}
$$




$$
=\omega_{2}\left(\varphi_{*}(\Delta)\right) \int_{E}\left\|u_{*}(s)-\tilde{u}(s)\right\| d s \leq 2 \omega_{2}\left(\varphi_{*}(\Delta)\right)[\mu(E)]^{\frac{p-1}{p}} r .
$$

From (4.14) and (4.20) we obtain that

$$
\begin{aligned}
\left\|x_{*}(\xi)-\tilde{x}(\xi)\right\| \leq & \frac{\lambda}{1-l_{0}} \int_{E}\left[l_{1}+l_{2}\|\tilde{u}(s)\|\right]\left\|x_{*}(s)-\tilde{x}(s)\right\| d s \\
& +\frac{2 \lambda}{1-l_{0}} \omega_{2}\left(\varphi_{*}(\Delta)\right)[\mu(E)]^{\frac{p-1}{p}} r .
\end{aligned}
$$

Condition $C$, Proposition 1, (2.2), (4.10) and (4.21) yield that

$$
\begin{gathered}
\left\|x_{*}(\xi)-\tilde{x}(\xi)\right\| \leq \frac{2 \lambda\left(1-l_{0}\right)^{-1} \omega_{2}\left(\varphi_{*}(\Delta)\right)[\mu(E)]^{\frac{p-1}{p}} r}{1-\lambda\left(1-l_{0}\right)^{-1}\left[l_{1} \mu(E)+l_{2}[\mu(E)]^{\frac{p-1}{p}} r\right]} \\
=\frac{2 \lambda \omega_{2}\left(\varphi_{*}(\Delta)\right)[\mu(E)]^{\frac{p-1}{p}} r}{1-l(\lambda)}=\beta_{*}(\Delta) .
\end{gathered}
$$

Since $\xi \in E$ is arbitrarily chosen, then the last inequality implies that

$$
\left\|x_{*}(\cdot)-\tilde{x}(\cdot)\right\|_{C} \leq \beta_{*}(\Delta) .
$$

Thus, for each $x_{*}(\cdot) \in \mathbf{X}_{p, r}^{H}$ there exists $\tilde{x}(\cdot) \in \mathbf{X}_{p, r}^{H, \Delta}$ such that the inequality (4.22) is verified. This means that

$$
\mathbf{X}_{p, r}^{H} \subset \mathbf{X}_{p, r}^{H, \Delta}+\beta_{*}(\Delta) B_{C}(1)
$$

Since $\mathbf{X}_{p, r}^{H, \Delta} \subset \mathbf{X}_{p, r}^{H}$, then (4.23) completes the proof.

\section{Piecewise constant control functions with norms from uniform mesh of $[\mathbf{0}, \mathbf{H}]$}

Let $\Gamma=\left\{E_{1}, E_{2}, \ldots, E_{N}\right\}$ be a $\Delta$-partition of compact set $E$ satisfying the relations (4.7)-(4.9), $\Lambda=\left\{0=w_{0}, w_{1}, \ldots, w_{a}=H\right\}(a \geq 1)$ be a uniform partition of the closed interval $[0, H]$, where $w_{j}=\frac{j}{a} H$ and $\delta=\frac{H}{a}$ is the diameter of the partition $\Lambda$. Define new set of control functions, setting

$$
\begin{gathered}
U_{p, r}^{H, \Delta, \delta}=\left\{u(\cdot) \in U_{p, r}^{H, \Delta}: u(\xi)=u_{i} \text { for every } \xi \in E_{i},\right. \\
\left.\left\|u_{i}\right\| \in \Lambda \text { for every } i=1,2, \ldots, N\right\} .
\end{gathered}
$$

It is obvious that $U_{p, r}^{H, \Delta, \delta} \subset U_{p, r}^{H, \Delta}$. The set of trajectories of the system (2.1) generated by the control functions $u(\cdot) \in U_{p, r}^{H, \Delta, \delta}$ is denoted by the symbol $\mathbf{X}_{p, r}^{H, \Delta, \delta}$. Denote

$$
\psi(\delta)=q_{*} \mu(E) \delta
$$

where $q_{*}>0$ is defined by $(2.9)$. 
Proposition 8. For every $H \in(0, \infty)$, finite $\Delta$-partition $\Gamma=\left\{E_{1}, \ldots, E_{N}\right\}$ of the compact set $E \subset \mathbb{R}^{k}$ and uniform partition $\Lambda=\left\{0=w_{0}, \ldots, w_{a}=H\right\}$ of the closed interval $[0, H]$ the inequality

$$
h_{C}\left(\mathbf{X}_{p, r}^{H, \Delta}, \mathbf{X}_{p, r}^{H, \Delta, \delta}\right) \leq \psi(\delta)
$$

is satisfied.

Proof. Let $\Gamma=\left\{E_{1}, E_{2}, \ldots, E_{N}\right\}$ be a finite $\Delta$-partition of the compact set $E \subset \mathbb{R}^{k}$, satisfying the relations (4.7)-(4.9) and let $x(\cdot) \in \mathbf{X}_{p, r}^{H, \Delta}$ be an arbitrarily chosen trajectory generated by the control function $u(\cdot) \in U_{p, r}^{H, \Delta}$. Since $u(\cdot) \in U_{p, r}^{H, \Delta}$, then

$$
\left\{\begin{array}{l}
u(\xi)=u_{i} \text { for every } \xi \in E_{i},\left\|u_{i}\right\| \leq H \text { for every } i=1,2, \ldots, N \\
\sum_{i=1}^{N} \mu\left(E_{i}\right)\left\|u_{i}\right\|^{p} \leq r^{p}
\end{array}\right.
$$

If $\left\|u_{i}\right\|<H$, then there exists $w_{j_{i}} \in \Lambda$ such that

$$
\left\|u_{i}\right\| \in\left[w_{j_{i}}, w_{j_{i}+1}\right) .
$$

Define new control function $u^{*}(\cdot): E \rightarrow \mathbb{R}^{m}$, setting

$$
u^{*}(\xi)= \begin{cases}\frac{u_{i}}{\left\|u_{i}\right\|} w_{j_{i}}, & \text { if } \quad 0<\left\|u_{i}\right\|<H \\ u_{i}, & \text { if } \quad\left\|u_{i}\right\|=0 \text { or }\left\|u_{i}\right\|=H\end{cases}
$$

where $\xi \in E_{i}, i=1,2, \ldots, N$ and $w_{j_{i}} \in \Lambda$ is defined by (5.3). It is obvious that the control function $u^{*}(\cdot)$ is piecewise constant and $\left\|u^{*}(\xi)\right\| \leq\|u(\xi)\|$ for every $\xi \in E$. From (5.2), (5.3) and (5.4) it follows that $u^{*}(\cdot) \in U_{p, r}^{H, \Delta, \delta}$ and

$$
\left\|u^{*}(\xi)-u(\xi)\right\| \leq \delta
$$

for every $\xi \in E$.

Now let $x^{*}(\cdot)$ be the trajectory, generated by the control function $u^{*}(\cdot) \in$ $U_{p, r}^{H, \Delta, \delta}$. Then $x^{*}(\cdot) \in \mathbf{X}_{p, r}^{H, \Delta, \delta}$ and (5.1), (5.5) and Proposition 5 yields that

$$
\left\|x^{*}(\xi)-x(\xi)\right\| \leq q_{*} \mu(E) \delta=\psi(\delta)
$$

for every $\xi \in E$, and hence

$$
\left\|x^{*}(\cdot)-x(\cdot)\right\|_{C} \leq \psi(\delta) .
$$

Thus, we conclude that for an arbitrarily chosen $x(\cdot) \in \mathbf{X}_{p, r}^{H, \Delta}$ there exists $x^{*}(\cdot) \in \mathbf{X}_{p, r}^{H, \Delta, \delta}$ such that the inequality (5.6) holds. This means that

$$
\mathbf{X}_{p, r}^{H, \Delta} \subset \mathbf{X}_{p, r}^{H, \Delta, \delta}+\psi(\delta) B_{C}(1) .
$$

Since $\mathbf{X}_{p, r}^{H, \Delta, \delta} \subset \mathbf{X}_{p, r}^{H, \Delta}$, then from (5.7) we obtain the proof. 


\section{Finite number of trajectories}

Let $\sigma>0, S=\left\{u \in \mathbb{R}^{m}:\|u\|=1\right\}$ and $S_{\sigma}=\left\{z_{1}, z_{2}, \ldots, z_{c}\right\}$ be a finite $\sigma$-net on $S$ and

$$
\begin{aligned}
U_{p, r}^{H, \Delta, \delta, \sigma}= & \left\{u(\cdot) \in U_{p, r}^{H, \Delta, \delta}: u(\xi)=w_{j_{i}} z_{l_{i}} \text { for every } \xi \in E_{i},\right. \\
& \text { where } \left.w_{j_{i}} \in \Lambda, z_{l_{i}} \in S_{\sigma}, i=1,2, \ldots, N\right\},
\end{aligned}
$$

where $\Delta$ is the diameter of the finite partition $\Gamma=\left\{E_{1}, E_{2}, \ldots, E_{N}\right\}$ of the compact set $E \subset \mathbb{R}^{k}, \delta$ is the diameter of the uniform partition $\Lambda=\{0=$ $\left.w_{0}, w_{1}, \ldots, w_{a}=H\right\}$ of the closed interval $[0, H]$. Note, that the set of control functions $U_{p, r}^{H, \Delta, \delta, \sigma}$ can be redefined as

$$
\begin{aligned}
U_{p, r}^{H, \Delta, \delta, \sigma}= & \left\{u(\cdot) \in L_{p}\left(E ; \mathbb{R}^{m}\right): u(\xi)=w_{j_{i}} z_{l_{i}} \text { for every } \xi \in E_{i},\right. \\
& \text { where } \left.w_{j_{i}} \in \Lambda, z_{l_{i}} \in S_{\sigma}, i=1,2, \ldots, N, \sum_{i=1}^{N} \mu\left(E_{i}\right) w_{j_{i}}^{p} \leq r^{p}\right\} .
\end{aligned}
$$

The set of trajectories of the system (2.1) generated by the control functions $u(\cdot) \in U_{p, r}^{H, \Delta, \delta, \sigma}$ is denoted by the symbol $\mathbf{X}_{p, r}^{H, \Delta, \delta, \sigma}$.

Since the set $U_{p, r}^{H, \Delta, \delta, \sigma}$ consists of a finite number control functions, then the set $\mathbf{X}_{p, r}^{H, \Delta, \delta, \sigma}$ includes a finite number trajectories. We also set

$$
\rho(H, \sigma)=q_{*} \mu(E) \sigma H,
$$

where $q_{*}$ is defined by $(2.9)$.

Proposition 9. For every $H \in(0, \infty)$, finite $\Delta$-partition $\Gamma=\left\{E_{1}, E_{2}, \ldots\right.$, $\left.E_{N}\right\}$ of the compact set $E \subset \mathbb{R}^{k}$, uniform partition $\Lambda=\left\{0=w_{0}, w_{1}, \ldots, w_{a}=\right.$ $H\}$ of the closed interval $[0, H]$ and $\sigma>0$ the inequality

$$
h_{C}\left(\mathbf{X}_{p, r}^{H, \Delta, \delta}, \mathbf{X}_{p, r}^{H, \Delta, \delta, \sigma}\right) \leq \rho(H, \sigma)
$$

is satisfied.

Proof. Let us choose an arbitrary trajectory $x(\cdot) \in \mathbf{X}_{p, r}^{H, \Delta, \delta}$ generated by the control function $u(\cdot) \in U_{p, r}^{H, \Delta, \delta}$. Inclusion $u(\cdot) \in U_{p, r}^{H, \Delta, \delta}$ implies, that

$$
\left\{\begin{array}{l}
u(\xi)=u_{i} \text { for every } \xi \in E_{i}, \quad\left\|u_{i}\right\| \leq H \text { for every } i=1,2, \ldots, N, \\
\left\|u_{i}\right\|=w_{j_{i}} \in \Lambda \text { for every } i=1,2, \ldots, N, \quad \sum_{i=1}^{N} \mu\left(E_{i}\right) w_{j_{i}}^{p} \leq r^{p}
\end{array}\right.
$$

By virtue of (6.2), there exists $y_{i} \in S, i=1,2, \ldots, N$, such that

$$
u(\xi)=w_{j_{i}} y_{i} \text { for every } \xi \in E_{i}, i=1,2, \ldots, N .
$$

Since $S_{\sigma}$ is a finite $\sigma$-net on $S$, then for each $y_{i} \in S$ there exists $z_{j_{i}} \in S_{\sigma}$ such that

$$
\left\|y_{i}-z_{j_{i}}\right\| \leq \sigma, i=1,2, \ldots, N
$$


Define new control function $u^{0}(\cdot): E \rightarrow \mathbb{R}^{m}$, where

$$
u^{0}(\xi)=w_{j_{i}} z_{j_{i}} \text { for every } \xi \in E_{i}, i=1,2, \ldots, N .
$$

It is not difficult to verify that $u^{0}(\cdot) \in U_{p, r}^{H, \Delta, \delta, \sigma}$ and from (6.2)-(6.5) we obtain that

$$
\left\|u(\xi)-u^{0}(\xi)\right\| \leq \sigma H
$$

for every $\xi \in E$.

Let $x^{0}(\cdot): E \rightarrow \mathbb{R}^{n}$ be the trajectory of the system (2.1) generated by the control function $u^{0}(\cdot) \in U_{p, r}^{H, \Delta, \delta, \sigma}$. Then $x^{0}(\cdot) \in \mathbf{X}_{p, r}^{H, \Delta, \delta, \sigma}$ and (6.1), (6.6) and Proposition 5 yield, that

$$
\left\|x(\xi)-x^{0}(\xi)\right\| \leq q_{*} \mu(E) \sigma H=\rho(H, \sigma)
$$

for every $\xi \in E$, and hence

$$
\left\|x(\cdot)-x^{0}(\cdot)\right\|_{C} \leq \rho(H, \sigma) .
$$

So, we have proved that for each $x(\cdot) \in \mathbf{X}_{p, r}^{H, \Delta, \delta}$ there exists $x^{0}(\cdot) \in \mathbf{X}_{p, r}^{H, \Delta, \delta, \sigma}$ such that the inequality (6.7) is held. This means that

$$
\mathbf{X}_{p, r}^{H, \Delta, \delta, \sigma} \subset \mathbf{X}_{p, r}^{H, \Delta, \delta}+\rho(H, \sigma) B_{C}(1) .
$$

Since $\mathbf{X}_{p, r}^{H, \Delta, \delta, \sigma} \subset \mathbf{X}_{p, r}^{H, \Delta, \delta}$, then inclusion (6.8) completes the proof.

\section{Main result}

From Propositions 6-9 it follows validity of the following theorem.

Theorem 1. For every $H \in(0, \infty)$, finite $\Delta$-partition $\Gamma=\left\{E_{1}, E_{2}, \ldots, E_{N}\right\}$ of the compact set $E \subset \mathbb{R}^{k}$, uniform partition $\Lambda=\left\{0=w_{0}, w_{1}, \ldots, w_{a}=H\right\}$ of the closed interval $[0, H]$ and $\sigma>0$ the inequality

$$
h_{C}\left(\mathbf{X}_{p, r}, \mathbf{X}_{p, r}^{H, \Delta, \delta, \sigma}\right) \leq \frac{\kappa_{*}}{H^{p-1}}+\beta_{*}(\Delta)+\psi(\delta)+\rho(H, \sigma)
$$

is satisfied, where $\Delta>0$ is the diameter of the finite partition $\Gamma=\left\{E_{1}, E_{2}, \ldots\right.$, $\left.E_{N}\right\}, \delta>0$ is diameter of the uniform partition $\Lambda, \kappa_{*}$ is defined by (3.1), $\beta_{*}(\Delta)$ is defined by (4.10), $\psi(\delta)$ is defined by (5.1), $\rho(H, \sigma)$ is defined by (6.1).

Theorem 1 implies the validity of the following corollary.

Corollary 1. For each $\varepsilon>0$ there exist $H(\varepsilon)>0, \Delta_{1}(\varepsilon)>0, \delta_{1}(\varepsilon)>0$ and $\sigma_{*}(\varepsilon, H(\varepsilon))>0$ such that for every $\Delta \in\left(0, \Delta_{1}(\varepsilon)\right), \delta \in\left(0, \delta_{1}(\varepsilon)\right)$ and $\sigma \in$ $\left(0, \sigma_{*}(\varepsilon, H(\varepsilon))\right)$ the inequality $h_{C}\left(\mathbf{X}_{p, r}, \mathbf{X}_{p, r}^{H(\varepsilon), \Delta, \delta, \sigma}\right) \leq \varepsilon$ is satisfied.

Remark 1 . Theorem 1 and Corollary 1 are valid for the sets $\mathbf{X}_{p, r}(\xi) \subset \mathbb{R}^{n}$ and $\mathbf{X}_{p, r}^{H(\varepsilon), \Delta, \delta, \sigma}(\xi) \subset \mathbb{R}^{n}$ for every $\xi \in E$, where

$$
\begin{aligned}
& \mathbf{X}_{p, r}(\xi)=\left\{x(\xi) \in \mathbb{R}^{n}: x(\cdot) \in \mathbf{X}_{p, r}\right\} \\
& \mathbf{X}_{p, r}^{H(\varepsilon), \Delta, \delta, \sigma}(\xi)=\left\{x(\xi) \in \mathbb{R}^{n}: x(\cdot) \in \mathbf{X}_{p, r}^{H(\varepsilon), \Delta, \delta, \sigma}\right\} .
\end{aligned}
$$




\section{Conclusions}

Approximation of the set of trajectories of the control system described by Urysohn type integral equation is discussed. The control functions are chosen from the closed ball of the space $L_{p}\left(E ; \mathbb{R}^{m}\right), p>1$, centered at the origin. In general, these kind of controls arise in the systems where the control resources are exhausted by consumption. The estimation for Hausdorff distance between the set of trajectories and its approximation depending of the discretisation and system's parameters is given. The presented result can be used for approximate calculation of the set of trajectories of the control systems arising in different problems of theory and applications. In particular, the obtained result can be applied for approximate calculation of the image of the closed balls of the space $L_{p}\left(E ; \mathbb{R}^{m}\right), p>1$, under Fredholm operators with continuous and Lebesgue measurable kernels.

\section{References}

[1] T. S. Angell, R. K. George and J. P. Sharma. Controllability of Urysohn integral inclusions of Volterra type. Electron. J. Diff. Equat., 79:1-12, 2010.

[2] J. Appell, A. Carbone and P. P. Zabrejko. Kantorovich majorants for nonlinear operators and applications to Urysohn integral equations. Rend. Mat. Appl., 12:675-688, 1992.

[3] E. J. Balder. On existence problems for the optimal control of certain nonlinear integral equations of Urysohn type. J. Optim. Theory Appl., 42:447-465, 1984.

[4] M. L. Bennati. An existence theorem for optimal controls of systems defined by Urysohn integral equations. Ann. Mat. Pura Appl., 121:187-197, 1979.

[5] F. Brauer. On a nonlinear integral equation for population growth problems. SIAM J. Math. Anal., 69:312-317, 1975.

[6] D. A. Carlson. An elementary proof of the maximum principle for optimal control problems governed by a Volterra integral equation. J. Optim. Theory Appl., 54:43-61, 1987.

[7] A. G. Chentsov. Approximative realization of integral constraints and generalized constructions in the class of vector finitely additive measures. Proc. Steklov Inst. Math., Suppl.2:S10-S60, 2002.

[8] R. Conti. Problemi di Controllo e di Controllo Ottimale. UTET, Torino, 1974.

[9] C. Corduneanu. Integral Equations and Applications. Cambridge University Press, Cambridge, 1991.

[10] K. G. Guseinov, A. A. Neznakhin and V. N. Ushakov. Approximate construction of reachable sets of control systems with integral constraints on the control. $J$. Appl. Math. Mech., 63:557-567, 1999.

[11] M. I. Gusev and I.V. Zykov. On extremal properties of the boundary points of reachable sets for control systems with integral constraints. Tr. Inst. Math. Mekh. UrO RAN, 23(1):103-115, 2017.

[12] W. Heisenberg. Physics and Philosophy. The Revolution in Modern Science. George Allen and Unwin, London, 1958.

[13] D. Hilbert. Grundzuge Einer Allgemeinen Theorie der Linearen Integralgleichungen. Druck und Verlag von B.G.Teubner, Leipzig und Berlin, 1912. 
[14] A. Huseyin and N. Huseyin. Dependence on the parameters of the set of trajectories of the control system described by a nonlinear Volterra integral equation. Appl. Math., 59:303-317, 2014.

[15] A. Huseyin, N. Huseyin and Kh. G. Guseinov. Approximation of the sections of the set of trajectories of the control system described by a nonlinear Volterra integral equation. Math. Model. Anal., 20:502-515, 2015.

[16] N. Huseyin. Compactness of the set of trajectories of the control system described by a Urysohn type integral equation. Int. J. Optim. Control Theory Appl. (IJOCTA), 7:59-65, 2017.

[17] N. Huseyin, Kh. G. Guseinov and V. N. Ushakov. Approximate construction of the set of trajectories of the control system described by a Volterra integral equation. Math. Nachr., 288:1891-1899, 2015.

[18] G. Ibragimov, A. Rakhmanov, I. A. Alias and A. M. J. Mai Zurwatul. The soft landing problem for an infinite system of second order differential equations. Numerical Algebra, Control and Optimization, 7:89-94, 2017.

[19] M. A. Krasnoselskii and S. G. Krein. On the principle of averaging in nonlinear mechanics. Uspekhi Mat. Nauk., 10:147-153, 1955.

[20] N. N. Krasovskii. Theory of Control of Motion: Linear Systems. Nauka, Moscow, 1968.

[21] A. R. Matviichuk and V. N. Ushakov. On the construction of resolving controls in control problems with phase constraints. J. Comput. Syst. Sci. Int., 45(1):1-16, 2006.

[22] B. T. Polyak. Convexity of the reachable set of nonlinear systems under $L_{2}$ bounded controls. Dyn. Contin. Discrete Impuls. Syst. Ser. A Math. Anal., 11(2-3):255-267, 2004.

[23] N. N. Subbotina and A. I. Subbotin. Alternative for the encounter-evasion differential game with constraints on the momenta of the players controls. J. Appl. Math. Mech., 39:376-385, 1975.

[24] P. S. Urysohn. On a type of nonlinear integral equation. Mat. Sb., 31:236-255, 1923.

[25] E. Vainikko and G. Vainikko. Product quasi-interpolation in logarithmically singular integral equations. Math. Model. Anal., 17(5):696-714, 2012. 